\title{
BACKGROUND RADIATION: PROBES AND FUTURE TESTS
}

\author{
MARTIN J. REES \\ Institute of Astronomy \\ Madingley Road \\ Cambridge, CBз OHA.
}

\section{The 'Standard' Hot Big Bang}

The clearest evidence for the 'hot big bang' is of course the microwave background radiation. Its spectrum is now known, from the FIRAS experiment on COBE, to be a very precise black body - indeed, the deviations due to high-z activity, hot intergalactic gas, etc are smaller than many people might have expected. Also the light element abundances have remained concordant with the predictions of big bang nucleosynthesis, thereby giving us confidence in extrapolating back to when the universe was a few seconds old (see Copi, Schramm and Turner 1994 for a recent review). These developments give us grounds for greater confidence in this model than would have been warranted ten years ago. Several things could have happened which would have refuted the picture, but they haven't happened. For instance:

(i) Objects could have been found where the helium abundance was far below 23 per cent.

(ii) The background spectrum at millimetre wavelengths could have been weaker than a black body with temperature chosen to fit the Rayleigh-Jeans part of the spectrum.

(iii) A stable neutrino might have been discovered in the mass range $100 \mathrm{eV}-1 \mathrm{MeV}$.

The key features that determine the present universe - the baryon/photon ratio, the fluctuations, etc - are legacies of exotic physics at ultra-early eras. These issues are now coming into sharper focus. However any inferences about the first microsecond remain tentative because the basic physics is itself uncertain. It is only when the universe has cooled down below $100 \mathrm{Mev}$ that 'conventional' physics becomes adequate, and we can have confidence in quantitative models. We have, however, heard at this meeting about one detailed alternative to the standard picture of the microwave background and nucleosynthesis. This is the model developed by Burbidge, Hoyle and Narlikar (these proceedings). I'd like to mention how measurements of anisotropies in the microwave background might help to distinguish this model from the 'standard' hot big bang. 
It is a generic feature of all models which attribute the microwave background to a dense big bang that the dominant opacity on the last scattering surface would be electron scattering. This means that the last scattering surface is located at the same redshift, and has the same thickness, whatever microwave observing frequency is used. Any angular fluctuations attributed to a 'last scattering surface' at high redshift should be the same at each frequency: if a strip of sky were scanned at two frequencies, the temperature fluctuations would track each other closely. (On the Rayleigh-Jeans part of the spectrum this is still true even when there is a Sunyaev-Zeldovich contribution). But in the model of Burbidge et al., the relevant opacity (due to carbon 'whiskers' etc) depends strongly on frequency. Scans at different frequencies are therefore probing 'surfaces' at different distances. One would therefore not expect the same fluctuations, except maybe on the very largest angular scales.

\section{Dark Matter}

Intimations of dark matter date back to studies of motions in clusters of galaxies in the 1930s; to analyses of motions within the local group, particularly the classic 1959 paper of Kahn and Woltjer; and to radio and optical studies of rotation in the outer parts of disc galaxies. This is not primarily a historical review, but I would like nevertheless to go back 20 years, to 1974 , because it was in that year that a consensus about the existence of dark matter was crystalised, particularly in two important papers. One of the classic papers, by Einasto, Kaasik, and Saar (1974), stated that "the mass of galactic coronae exceeds the mass of populations of known stars by 1 order of magnitude, as do the effective dimensions. The mass luminosity ratio rises to $f=100$ for spiral and $f=120$ for elliptical galaxies. With $H=50 \mathrm{~km} / \mathrm{sec} / \mathrm{Mpc}$ this ratio for the Coma cluster is 170 ". In the second paper, by Ostriker, Peebles, and Yahil (1974), it was stated that "currently available observations strongly indicate that the mass of spiral galaxies increases almost linearly with radius to nearly $1 \mathrm{Mpc}$, and that the ratio of this mass to the light within the Holmberg radius is $200 M_{\odot} / L_{\odot} "$.

These particular inferences have been buttressed enormously by progress in the last 20 years; but it is remarkable that the conclusions have not be drastically changed.

Another indirect constraint on the amount of dark matter in baryonic form comes from the abundances of light elements predicted by cosmic nucleosynthesis. As has been well-known since the late $1960 \mathrm{~s}$, these abundances depend on the baryon density when the universe cools through the temperature range from $1 \mathrm{Mev}$ to $100 \mathrm{keV}$, and therefore (since the present background temperature is known) can be related directly to the present baryon density. The predicted helium abundance increases only slowly with density, but the measurements of helium are now precise enough to provide a significant upper limit. Deuterium, however, is a more sensitive measure of the primordial baryon density. Since it is an intermediate product in the production of helium, more deuterium survives in a universe of low baryon density. Moreover, it is now much more clearer than it was in the 1960 s that deuterium is best explained as a relic of the early universe.

1974, plainly a vintage year for this subject, also saw the publication of a review by Gott, Gunn, Schramm, and Tinsley (1974). These authors adopted a synoptic approach, 
and tried to seek consistent ranges for the density parameter $\Omega$ and the Hubble constant. They considered three constraints. The first was the requirement that the universe (whose age depends on the Hubble constant and, in Friedman models, on $\Omega$ ) should be older than the oldest stars. The second was an upper limit on the baryon density from deuterium. And the third was a lower limit to $\Omega$, of order 0.1 , set by the amount of dark matter that was reliably established by dynamical arguments. They claimed that there was a very small window, with $\Omega$ of order 0.1 and a low Hubble constant, such that the age constraints could be satisfied and all the reliably-established dark matter could be baryonic.

How have Gott et al.'s arguments fared in the last twenty years? First, the uncertainties about the Hubble constant and stellar ages are still with us. So let us confront that problem squarely and pass on.

Much more is known about the amount of dark matter in clusters of galaxies, though the net effect of the newer evidence does not substantially change the old estimates. However, the issue of extra dark matter between clusters, maybe even sufficient to provide the critical density, is now a more lively one, and I shall return to it later.

Estimates of deuterium as a measure of baryon density have improved, particularly through a better understanding of the relationship of deuterium and helium ${ }^{3}$. There has recently been a flurry of interest in cosmic deuterium, stimulated by the claim of a high relative abundance of deuterium to hydrogen, of order $3 \times 10^{-4}$, in a high redshift damped Lyman-alpha absorption system along the line of sight to a quasar (Songalia et al. 1994). If this result were to stand up, it would push down the permitted baryon density, completely ruling out the possibility that most halo dark matter could be baryonic unless one abandons other standard assumptions. However, it would be wise to suspend judgement on this issue. The alleged deuterium line is a weak satellite of a very strong feature attributed to highcolumn-density HI. It is indeed displaced by 80 kilometres per second, equivalent to the expected isotopic shift, from the centre of a strong hydrogen feature, and there is only a few per cent chance of finding a random weak line in the Lyman forest in this position. But there may very well be an excess of weak 'satellite' lines close to any damped Lyman alpha system (due to gas associated with the same 'protogalaxy'). Until we are sure that there are more systems displaced by $80 \mathrm{~km} / \mathrm{s}$ than by, say, 60 or $100 \mathrm{~km} / \mathrm{s}$ the significance of this claim for high deuterium must remain in doubt. Further data, particularly from the Keck Telescope, ought to settle this question within the next couple of years.

The possibility of baryonic dark matter in stars or stellar remnants was addressed with particular thoroughness by Carr, Bond, and Arnett (1984). These authors showed, through a variety of arguments that are now well-known, that there were two possible mass ranges. Dark matter could exist in black holes in the mass range between a few hundred and $10^{6}$ solar masses, which could be a remnant of a population of early massive stars that ended their lives collapsing via the pair production instability. The other possibility is brown dwarf or planetary mass objects, similar to stars except they are below the threshold of around 0.07 solar masses needed to trigger hydrogen fusion. Some constraints on high mass objects in our Galaxy are set by the lack of evidence for accretion onto those passing through the Disc, and so forth. But the most interesting recent work, involving gravitational microlensing and the search for evidence of lensing by low-mass compact objects in our own galaxy, features strongly in another symposium being held in parallel with ours, so I shall say no more about it here. 
Traditionally, the dark matter in clusters has been inferred from application of the virial theorem to galaxy motions. But there are now two other lines of attack. Maps of the X-ray brightness profile and temperature are now good enough to allow estimates of the depth of the gravitational well confining the hot X-ray emitting gas. And the detection of large numbers of very faint background galaxies whose shapes are distorted, often into conspicuous arcs, by the effects of light bending due to the clusters gravitational field, will soon offer very direct information about the total mass distribution, whatever that mass may be. One of the early highlights of the data from the post-refurbishment HST is a superb picture of the cluster Abell 2218, with a redshift 0.18, by Ellis, Kneib, and Smail (1994), which shows very large numbers of obvious background arcs.

It will soon seem natural to discuss the structure and dynamics of cluster masses in an order different from the traditional one. We shall first infer the depth of the potential well directly by reconstructing it from the observed distortion by gravitational lensing of background galaxies. It will then be possible to decide whether the observed spatial distribution of galaxies, and the spread in their velocities is consistent with an isotropic equilibrium in that particular potential; if it isn't, the angular distribution of velocities must otherwise be more complex, or the system must be out of equilibrium. X-ray maps will reveal whether the gas has a temperature and density profile consistent with that potential. If it isn't, we shall be motivated to consider whether the gas is partially supported by rotation, macroscopic bulk motion, magnetic pressure, relativistic particles, etc. (The gas can of course be somewhat inhomogeneous, but the clumping factor is constrained because gas confined in the potential well cannot be on a very much higher adiabat than the gas that dominates the X-ray emission.)

Of course clustering must be seen in the more general context of overall cosmic structure formation. Numerical simulations of this are now a heavy industry, and an increasingly sophisticated one. Most of these simulations are based on the assumption that the dominant gravitating stuff is nonbaryonic. So let us briefly consider this option.

One of the main changes since Gott et al. wrote their 1974 paper has been the much greater willingness to invoke nonbaryonic matter. Non-zero neutrino masses are no longer thought theoretically unacceptable, and there is a willingness to invoke new kinds of particles, particularly those predicted by supersymmetric theories. What are the prospects for direct detection of nonbaryonic matter? Neutrinos seem impossible to detect by feasible current techniques, and axions present a very severe experimental challenge. But there has been substantial interest in detecting heavy neutral particles, such as the lightest stable supersymmetric particles. These techniques involve detecting the recoil in the rare event when one of these particles, which would pervade the entire halo moving with speeds of about $10^{-3} c$, interacts with a nucleus in an experimental detector. We should certainly spare a thought, and give every possible encouragement, to those of our colleagues, mainly working down mineshafts, who have accepted the challenge to detect dark matter. Even the optimist cannot predict success with great confidence, but the attainable upper limits are themselves becoming significant, and detection of such particles would tell us what 90 per cent of the Universe is made of, as well as perhaps discovering an entirely new class of particles that cannot be produced terrestrially.

Apart from direct detection, another way of reducing the range of non-baryonic options for the dark matter would be by progress in particle physics. If we knew what particles 
should exist in the ultra-early universe, together with their masses and annihilation crosssections, it should be possible to calculate which (if any) survive in sufficient numbers to contribute to the dark matter, with the same confidence that we can now apply to calculations of primordial nucleosynthesis. Even in the absence of such direct knowledge, something can be learnt about non-baryonic dark matter by exploring its implications for cosmogony.

\section{The 'Cold Dark Matter' Model}

The most intensively studied model for structure formation involves the hypothesis that the dark matter is 'cold', in the sense that its thermal motions are never sufficient to smear out small-scale structure. A particular benchmark for comparison of observations has been the so-called 'standard' CDM model. This model involves a package of five assumptions.

1. The primordial spectrum has the Harrison-Zeldovich form, and the fluctuations are Gaussian.

2. The universe is dynamically dominated by cold nonbaryonic matter which interacts only gravitationally with everything else.

3. The density is taken to be equal to the critical value, in other words, $\Omega=1$.

4. Galaxies are related to dark matter by a simple biasing prescription.

5. Neutrino masses are taken to be zero.

The outcome of these simulations is tested against the data by comparing the relative amplitude of clustering on different scales with what is actually observed at the current epoch. The z-dependence of the structure offers another test. There is limited evidence on how the observed large-scale structure has evolved, but on galactic scales there are constraints back to redshifts of 5 from quasars, neutral hydrogen clouds, etc.

It is now well-known that this 5 -item package, the 'standard' CDM model, runs into some problems with reconciling small- and large-scale structure and the microwave background fluctuation amplitude. However, this doesn't mean that the dark matter cannot be in the form of 'cold' non-baryonic matter, because there are a number of modifications of the other four hypotheses which are physically motivated and by no means simply 'ad hoc'. First, the primordial fluctuation spectrum could be tilted, so that the amplitude increases slowly with scale. Indeed most inflationary models predict that this should occur. Also one could consider models where $\Omega$ is different from unity: these are either open or else flat with the extra curvature made up by a non-zero cosmological constant. Another uncertainty concerns the relation between the galaxies and the dark matter. The simple scheme which depicts the biasing in terms of one parameter is certainly oversimplified: the galaxy formation efficiency may depend on environment, etc., in many ways. It may be easier to test the models by directly probing the distribution of the dark matter, either by determining the motions it induces in the galaxies which deviate them from the Hubble flow, or by detecting weak lensing due to inhomogeneities on supercluster scales. 
And it may turn out that neutrino masses are not exactly zero. The so-called 'hybrid' or 'mixed' dark matter models, in which a neutrino has a mass of a few eV, surmount some of the difficulties of standard CDM. If experimentalists find such evidence for neutrino masses, believers in CDM would delightedly incorporate it in their existing models, ending up with a better fit.

\section{Is $\Omega=1$ ?}

The other change since Gott et al.'s classic paper is that there is now a strong theoretical prejudice in favour of $\Omega=1$, stemming from the attractiveness of the general concept of an inflationary universe. Such models naturally predict that the universe expands enough to stretch the universe flat, in the sense that the Robertson-Walker curvature radius would become enormously larger than the present Hubble scale. Anything different from a flat universe would, as is well known, involve fine tuning in the expansion factor. This tuning is implausible at the level of a few per cent, even in the more optimistically contrived scenarios. However, most variants of inflation allow an even stronger argument in favour of $\Omega=1$. In these models, if the universe had inflated only enough to make the present RobertsonWalker curvature of order the Hubble radius, there would be quadrupole or dipole effects in the microwave background of order unity. Some recently developed models, however, manage to avoid this latter constraint.

What, then, is the observational case for or against a critical density? I think everyone would agree that this is still tentative. Some of the classical 'geometrical' methods should soon become more helpful. The Hubble diagram for supernovae may be extended to high enough redshifts to reveal the deceleration parameter; further studies may firm up the earlier tentative evidence from the angular diameters of high redshift sources in favour of a high density. On the other hand, if the Hubble constant error bars are reduced, and the Hubble time becomes less than 15 billion years, this will obviously argue against a critical density in which the time since the big bang is only two thirds of the Hubble time.

I should like to conclude this section by mentioning some rather less direct lines of evidence on the density.

Clusters of galaxies offer two such arguments. The first is an inference from the irregular shapes of most clusters, indicating that they have undergone recent mergers of subcomponents each comprising a substantial fraction of the total mass. In a low density universe, structure forms early and is thereafter frozen in. On the other hand, formation continues if $\Omega$ is high. Therefore the prevalence of conspicuous substructure points towards a high $\Omega$, though I think this is not yet quantitative enough to allow us to say that it requires the full critical density.

But a quite distinct argument, again based on clusters, suggests a low $\Omega$. X-ray data show that the baryon fraction in a cluster, mainly in hot gas, is typically between 10 and 20 per cent. (The exact fraction depends, of course, on the Hubble constant). This has been inferred from detailed study of the Coma cluster (White et al.1993), and also, in a recent paper by White and Fabian (1994), for a sample of 19 clusters. When the baryonic fraction of the mass in the cluster is compared with the baryonic fraction in the universe allowed by standard big bang nucleosynthesis, there is a contradiction if $\Omega$ is more than about 0.3 . If 
$\Omega$ is indeed high, one has either to abandon standard nucleosynthesis, or understand how baryons can be segregated relative to dark matter (by a factor of about 3 ) even on scales as large as the turnaround radius of a cluster. It could be that the resolution of this dilemma will come from a combination of small effects and uncertainties, but at the moment it seems a serious argument against $\Omega$ of 1 .

Another quite different estimate of $\Omega$ will soon come from studies of microwave background fluctuations. The COBE data refer to angular scales of $10^{\circ}$. However, several other groups are now reporting fluctuations on angular scales of order $1^{\circ}$. These latter scales seem to display a larger amplitude than found by COBE. This is precisely what is expected if one is probing scales smaller than the horizon at recombination, because there is then a contribution from Doppler motions, etc. That angular scale is about $2^{\circ}$ in a flat model, but scales as $\Omega^{\frac{1}{2}}$. Firm evidence for an upturn in the background fluctuation amplitude on angular scales of one or two degrees would be hard to reconcile with a low $\Omega$, where any Doppler contribution would be restricted to angular scales below one degree.

\section{Origin of Magnetic Fields}

This symposium has dealt with background radiation in all wavebands. At the risk of 'stretching' the definition of background radiation rather far, I'd like to conclude with some remarks on the zero-frequency (DC) limit - large-scale cosmic magnetic fields - whose origin is a mysterious and under-discussed aspect of cosmogony. Fuller details are given elsewhere (Rees 1994).

Cosmic magnetic fields probably owe their present pervasive strength to dynamo amplification. But there must then have been an initial seed field - otherwise the dynamo process would have had nothing to feed on. It seems to be generally 'taken for granted' that the requisite seed field will be there. In many astrophysical contexts this confidence may be justifiable: if the dynamical (and amplification) timescale is short enough, there can be a huge number of e-foldings; a merely infinitesimal statistical fluctuation might then suffice. But the large-scale fields in disc galaxies seem to pose a less trivial problem. The amplification timescale may be $2.10^{8}$ years; even by the present epoch there has been time for only 50 e-foldings. The galactic field could not, therefore, have built up to its observed strength by the present day, unless the seed were of order $10^{-20} G$ - very weak, but not infinitesimal. Moreover, if it turned out that substantial fields existed even in high-z galaxies whose discs may have only recently formed, the seed would need to have been correspondingly higher.

Star formation would proceed differently (with regard both to its rate, and the shape of the initial mass function) if there were no magnetic field: the field modifies the Jeans mass and contributes to transfer of angular momentum. So we cannot hope to model galactic evolution adequately without knowing when the field builds up to a dynamicallyimportant strength. (Moreover, even a weaker field may be significant through its influence on thermal conductivity, etc). If several galactic rotation periods elapsed before a dynamically-significant field built up, then the oldest stars may well, for this reason alone, have a different luminosity function.

There is as much reason to believe that the absence of a magnetic field affects the IMF as to believe that a lack of heavy elements does so (though the quantitative nature of the effect is as uncertain in the one case as the 
other).

Could a field even if only $10^{-20} G$, have been created in the early stages of the big bang? The ultra-early universe may have undergone a phase transition; and maybe this transition could (as in a cooling ferromagnetic material) spontaneously create a field. Because the relevant physics is exotic and poorly understood, we plainly cannot rule this possibility out. However, the correlation scale would be limited to the scale of the horizon. So, even if the field had a high local energy density, it would be primarily on such small scales that it would quickly decay, and there would be no chance of getting even $10^{-20} \mathrm{G}$ on the scale of a protogalaxy.

This is a generic problem with attributing a cosmological origin to the field, even if a convincing microphysical mechanism could be found. (Of course, this problem would be surmounted if there were an overall cosmic anisotropy).

A cosmic 'battery' mechanism would have to await nonlinearities that lead to shock waves or the formation of bound systems that exert tidal torques on each other. Compton drag can then (cf Zeldovich, Rosmaikin and Sokoloff, 1983) gradually build up a current in a rotating protogalaxy. If plasma moves at speed $V$ relative to the frame in which the microwave background is isotropic, its motion would be damped out on a timescale $\left(m_{p} / m_{e}\right) t_{c o m p}$, where $t_{c o m p}=m_{e} c / \sigma_{T}\left(a T^{4}\right)$ is the usual Compton cooling timescale for electrons. To couple electrons and ions, an E-field of strength $m_{e} V / e t_{c o m p}$ must maintain itself in the plasma. A protogalaxy of radius $\mathrm{R}$ rotating with speed $\mathrm{V}$ would be gradually braked by Compton drag, and the E field within it (with, of course, non-zero curl) would build up a B-field at a rate $\left(m_{e} c^{2} / e t_{c o m p}\right)(V / R)$. For a protogalaxy at redshift $z \simeq 5$, this process yields a field only of order $10^{-21} G$.

It is more promising to consider a later origin. I'd like briefly to mention two options: field generation by the first generation of stars, and also in radio galaxies.

Protostars condensing in the present-day interstellar medium start off with too much magnetic flux rather than too little. But the field in a star at the end of its life may be insensitive to the conditions at its birth: even if a star initially had zero field, the Biermann battery could generate a seed field, on which dynamo amplification (by a huge number of factors of $e$ if necessary) could operate. If such a star exploded as a supernova, then a wind spun off the remnant pulsar could pervade several cubic parsecs with a field of order $10^{-4} G$ (just as in the Crab Nebula). So the first few supernovae could have created a weak field throughout the galactic disc, even if a larger-scale battery hadn't already done so.

Provided that the large-scale modes could be preferentially amplified, these stellargenerated fields would be adequate seeds for a galactic dynamo. For a quantitative estimate, note that each hemisphere of the Crab Nebula contains an (equal and opposite) flux of order $10^{34} G \mathrm{~cm}^{2}$. If $\mathrm{N}$ similar remnants formed in, for instance, a young galactic disc, the net flux would then be larger by a factor $N^{x}$. The appropriate value for $x$ isn't obvious. The net effect depends on the two hemispheres evolving differently - otherwise the net flux cancels out. To assume that $x=1 / 2$ may therefore be over-optimistic. A better guess might be $x=1 / 3$. This is appropriate if the remnants are randomly oriented, and the galaxy can be modelled as the interior of a surface which slices a fraction $N$ of the remnants. As an example, if $N=10^{6}$, the large-scale component of the field in a protogalactic disc of $10 \mathrm{kpc}$ radius would be $3.10^{-8}-3.10^{-9} G$, for $x$ in the range $1 / 3-1 / 2$.

The highest-redshift radio galaxies formed when the formation of typical galaxies (espe- 
cially those with discs) still lay in the future. The fields in the lobes of radio galaxies could have been generated in the active nucleus of the associated galaxy and expelled along collimated jets (resembling a scaled-up and directional version of the relativistic pulsar wind that generates the Crab Nebula's field). In the nucleus itself, the dynamical timescale may be as short as a year, or even a few hours if the relevant processes occur close to a black hole. So we need not worry about what seeded the AGN itself: there is time for a battery process to operate, or even for a dynamo to be seeded by an infinitesimal field. Thus, a radio galaxy's field, like that in a supernova remnant, can be accounted for even if the progenitor central object had zero field when it formed.

Galaxies may acquire their discs at $z<2$ via collapse of a slowly-rotating cloud with turn-around radius $>50 \mathrm{kpc}$. If the infalling material had been 'contaminated' by a fraction $f$ of a radio source lobe, the large-scale component of the seed field would be $3.10^{-8}\left(f / 10^{-4}\right) G$. So only a small value of $f$ might suffice. However the seed fields in discs could only be attributed to early radio sources if the lobe material were subsequently mixed into a larger volume. This is because radio galaxies are relatively thinly spread through the universe, being far less common than disc galaxies.

The origin of the seed field for the galactic dynamo is a more challenging question than the seeding of smaller-scale cosmic dynamos because the galactic timescale is so long, and the amplification correspondingly slow. (And I have assumed, of course, that the galactic dynamo mechanism is indeed efficient - the problem is obviously far worse if it isn't.) There are as yet no firm grounds for expecting significant fields in the ultra-early universe - indeed there are good reasons for expecting the large-scale components of any such field to be uninterestingly small. And the galactic-scale batteries where Compton drag provides the emf would be barely enough to yield an adequate seed. More promising, in my view are supernova remnants from early stars, or the lobes of high-z radio galaxies, either of which could yield $\sim 10^{-9} G$.

These mechanisms are not mutually exclusive; and there are clearly strong inter-relations between fields in stars, in AGNs or radio galaxies, and in galactic discs. The build-up of a galactic magnetic field depends on how strong the seed field is and when it was generated. Because of the field's importance in star formation, we have little chance of really understanding what a high-redshift galaxy should look like until these issues have been given a good deal more attention by experts in cosmic magnetism.

\section{References}

Carr, B.J., Bond, J.R. and Arnett, W.D. 1984, Ap.J., 277, 455

Copi, E., Schramm, D.N. and Turner, M.S. 1994, Science (in press)

Einasto, J., Kaasik, A. and Saar, E. 1974, Nature, 250, 309

Ellis, R.S., Kneib, J.P. and Smail, I. 1994, Ap.J. (in press)

Gott, J.R., Gunn, J.E., Schramm, D.N. and Tinsley, B. 1974, Ap.J., 194, 543

Kahn, F.D. and Woltjer, L.W. 1959. Ap.J., 130, 703

Ostriker, J.P., Peebles, P.J.E. and Yahil, A. 1994, Ap.J. (Lett.), 193, L1

Rees, M.J. 1994 in "Cosmical Magnetism" ed. D. Lynden-Bell, p155 (Kluwer, Dordrecht)

Songalia, A., Cowie, L.L., Hogan, C.J. and Rugers, M. 1994, Nature, 368, 599 
White, D.A. and Fabian, A.C. 1994, MNRAS (in press)

White, S.D.M. et al. 1993, Nature, 366, 429

Zeldovich, Y.B., Ruzmaikin, A.A., and Sokolov, D.D. 1983, "Magnetic Fields in Astrophysics", Gordon and Breach. 